\title{
Calculated injection times of divided fuel dose in a compression ignition engine
}

This study presents analyses concerning control processes of injection times of divided dose of fuel, startning from setting of injection time in controller up to moment of execution in compression ignition engine. Multiple injection doses were analyzed taking into consideration both sums of individual portions of doses as well as gaps between the doses. Studies were performed for various configurations of the doses, with different values of injection times and gaps between the injections. Measurements were performed for three measuring groups containing single-, two-, and five-portion doses. At constant values of time in a given group it have been applied variable injection pressures. The most important factors determining division of fuel dose were discussed.

Key words: diesel engine, common rail injection system, indicator of injection, fuel injection rate, injection time

\section{Introduction}

Permanent strive for development of more and more perfect automotive combustion engines is caused by necessity of assurance of high efficiency, durability and compliance with increasingly stringent regulations on harmful exhaust emissions. Perfection of combustion process proves natural pursuance of development of more efficient and better-controlled systems of fuel supply. Use of suitable control algorithms and selection of appropriate injection times make it possible to achieve both required parameters of engine performance and compliance with legal standards concerning purity of exhaust gases.

Vast majority of Diesel engines manufactured nowadays is equipped with high-pressure Common Rail systems. The main advantage of such systems is ability of controlling in wide range of engine operation, such parameters as time and pressure of injection, as well as form of the injection. Knowledge of injection time is important, because during operation of injection system, amount of fuel supplied to cylinder of engine is changed basing on this value.

Analyzes of injection processes which are performed by fuel supply systems with Common Rail have showed significant differences between injection times preset by controller and times of the injection. The authors have undertaken attempt to assess these differences, their causes and directions of change.

Nowadays, issues related to the analysis of multiple injection rate are discussed in terms of the effect of individual injection parts on the injection process. These tasks are carried out both by means of model analyzes [1] and by experiments carried out on test beds [2]. Rarely you can see analyzes of the multiple injection rates, especially in terms of changes in both time and fuel mass delivered in the parts.

This paper is the third publication devoted to analysis of injection times of multiple fuel dose. In the first publication [3] are presented characteristic injection times in function of operational parameters of injection system, while in the second publication are discussed controlled injection times and times of needle lift [5].

\section{Scope of the analysis}

With fixed pressure values in fuel rail, injection times determine amounts of injected fuel and form of the injection.
These times, which were input to read-only memory of the controller of the engine (ECU unit), or to internal memory of experimental controller are called as preset times of injection. The concept of ,calculated times" was also introduced to the considerations. Under this concept is meant injection time of individual portion of dose and time of gap between the doses, determined on the basis of pressure increase in indicator of injected dose and on the basis of calculated courses of injection. With such approach it is necessary to use dependencies resulting from equation of mass conservation, needed to carry out approximations of measured courses and to take into consideration changes in parameters of injected fuel. These are not, therefore, the times obtained directly, for instance by measuring of needle lift, but obtained indirectly, based on recalculations with taking into consideration additional quantities. Calculated injection times are the response of the system to the preset times. Calculated injection times and characteristic parameters of fuel outflow rate from the injector directly result from determined courses of injections.

Thus, it is possible to determine relationships between preset, electric courses which control injectors in fuel supply system, and calculated injection times, reflecting changes in fuel stream supplied to cylinder. The comparison is especially significant in optimization of combustion process in cases where various forms of injection are used, and hence where divided injection doses are supplied. Therefore, the studies were undertaken to explain a reasons and to determine direction of changes of these quantities.

The objective of the study was to determine differences between preset times of injection and calculated times of injection, and hence, quantities necessary during development and modification of controlling algorithms to compression ignition engines. Proper selection of these quantities is necessary to ensure low operational fuel consumption. This knowledge is also useful for modeling of working cycle of compression ignition engine.

In turn, due to need to assure long operational life of vehicle with unchanged operational parameters, it is necessary that injection system should comply with high requirements, such as: stability of multiple doses supply, proper response of the system to preset, differentiated times, and unchanged accuracy of injection process during complete operational life of the system [8]. Assessment of 
dosing process is impossible without knowledge of preset times of injection and response to such enforcement, i.e. calculated injection times.

Considering the above, it was necessary to determine dependence between differences in the above mentioned injection times and pressure in fuel rail, and method of division of fuel dose into portions.

As already mentioned above, multiple doses of injection were analyzed. Therefore, in each measuring case the comparisons were performed in two ways:

- for sums of times of individual portions of dose, and

- for total injection times, i.e. for sum of times of individual portions of the doses and gaps between them.

The considerations were accomplished for various configurations of multiple doses, with different values of injection times and gaps. Measurements were performed for three measuring groups. The first group, with two-portion division of the dose, consisted of short, uniform values of the preset times and low, variable pressures of injection.

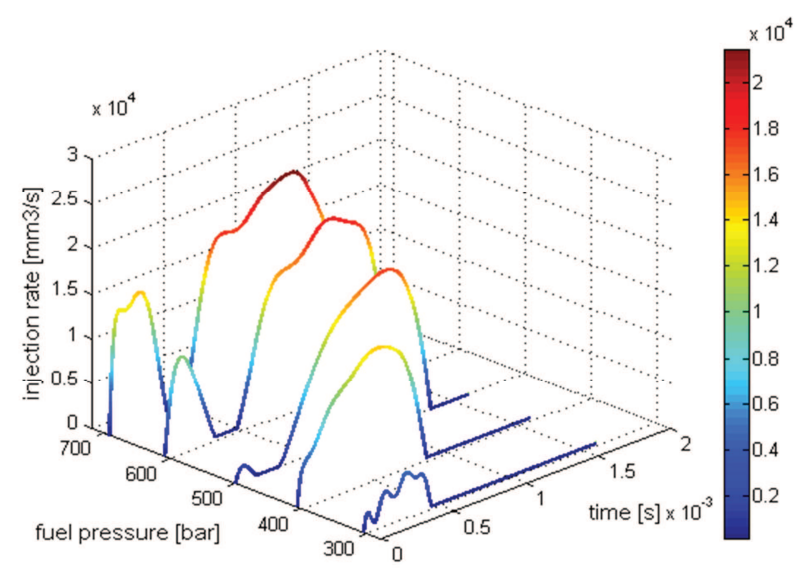

Fig. 1. Injection courses of two-portion dose (first measuring group)

The second group with values of different preset times, with three-, two-, and single-portion division and different pressures. In these cases, the aim was to obtain similar values of aggregated dose of the fuel $50 \mathrm{mg}$ /injection. Values measured in the test bed differed from assumed values from $-1.8 \%$ to $5.1 \%$.

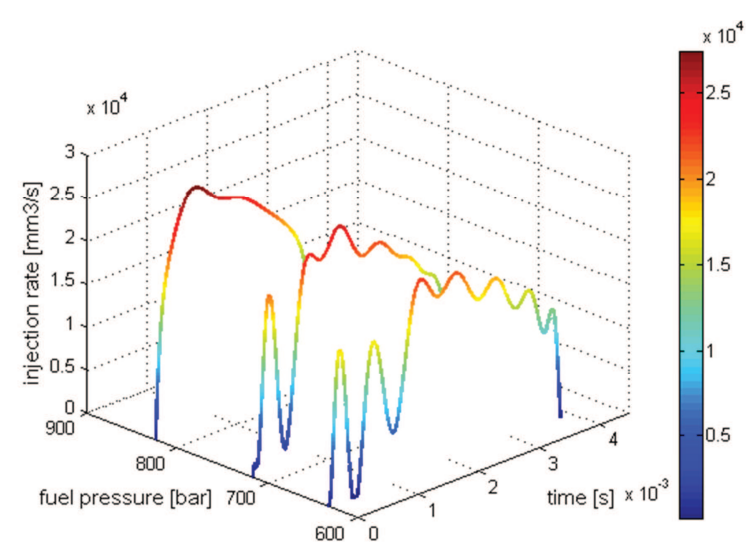

Fig. 2. Injection courses of the doses with constant value (second measuring group)
The third group consisted of identical values of times, preset for five-portion division and variable values of fuel pressure in the rail, selected from range of average values as used during operation of vehicles.

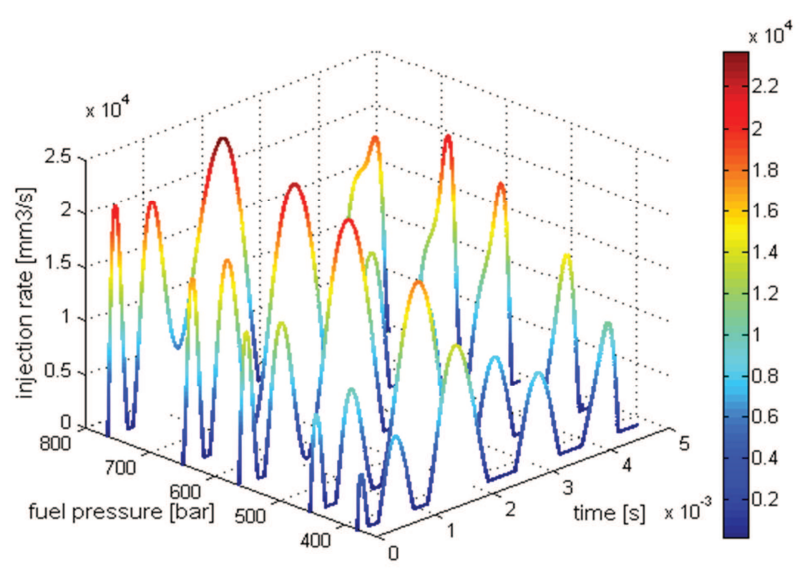

Fig. 3. Injection course of five-portion dose (third measuring group)

In turn, in the Fig. 4-6, for each from the groups, are presented ranges of the changes, both preset parameters and form of the injection.

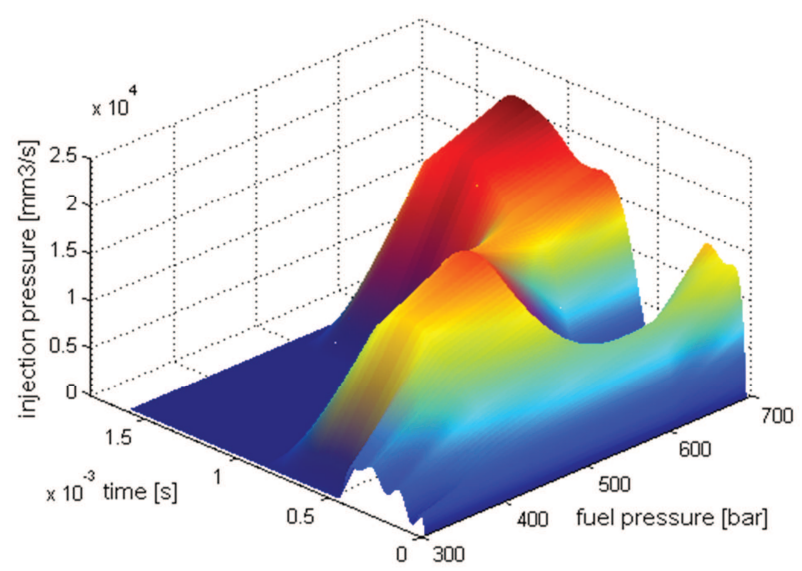

Fig. 4. Changes in parameters and forms of injection of two-portion dose (first measuring group)

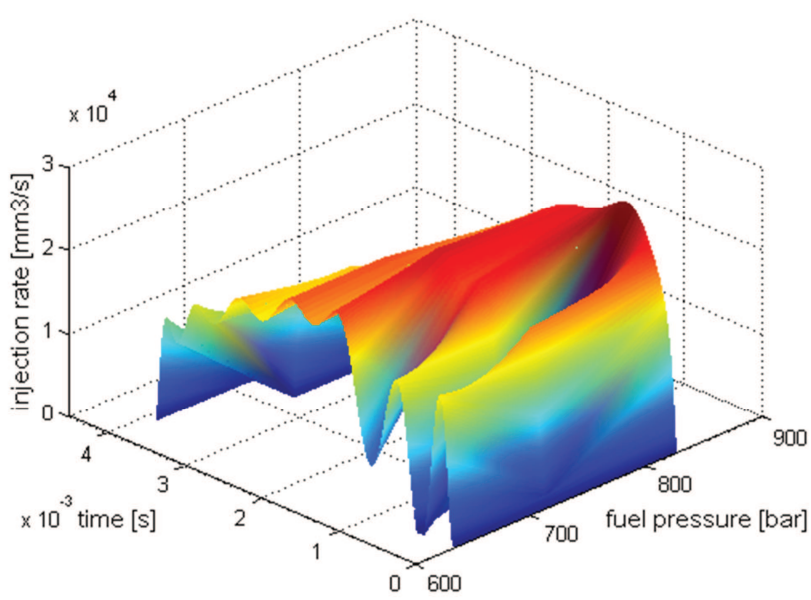

Fig. 5. Changes in parameters and forms of injection of the doses with constant value (second measuring group) 


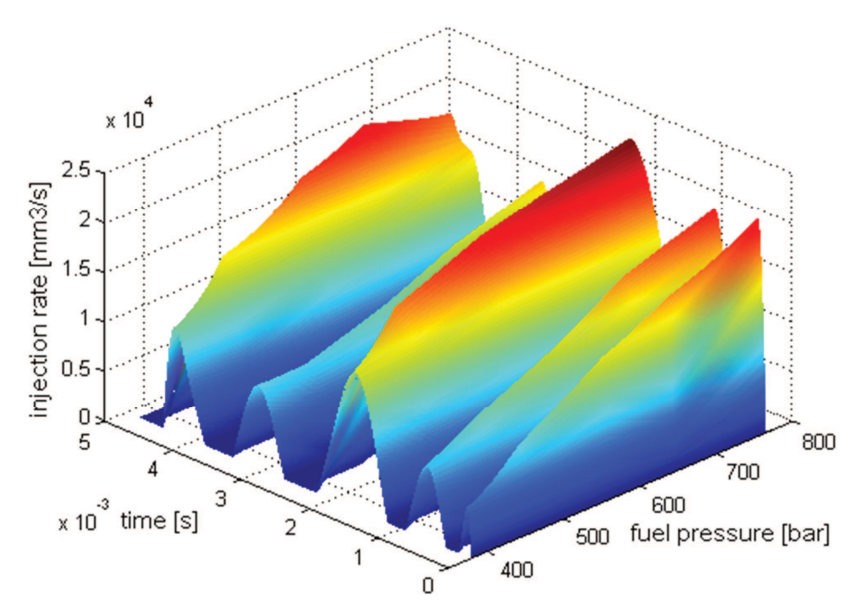

Fig. 6. Changes in parameters and forms of injection of five-portion dose (third measuring group)

Investigated injection system with Common Rail was controlled by KSSiP-2 controller, developed and produced in the Department of Combustion Engines and Vehicles [5]. Calculated injection times were determined based on courses of voltage change in piezo-quartz GU-21D transmitter produced by AVL, located in chamber of electronically controlled indicator of injection dose [6]. The voltage was measured at the output from the transmitter, using Tektronix TDS-3014 electronic oscilloscope. Based on measured voltage were evaluated calculated injection times and characteristic quantities connected with injection course of divided dose.

\section{Comparative analysis of preset and calculated injection times}

Injection time accomplished by injection system can be determined by various methods. To this purpose, in the study [4], effect of fuel injection in form of pressure growth in chamber of electronically controlled indicator of injection dose was used. Determined in such way courses were called as times of needle lift. Courses of pressure variations can be also used as a basis for calculations of injection course, and these in turn to evaluation of calculated injection times. It might seem that since comparisons of preset times and needle lift were carried out earlier [4], there is no needed to compare the preset times and calculated times. However, times of the lift and calculated times are different. Necessity of approximation of measured signals should be recognized as the most significant reason, because measured courses are not smooth, and this causes difficulties in calculations of derivative of pressure in function of time, needed to evaluation of calculated injection times. Sometimes there are also difficulties in evaluation of zero line of fuel outflow rate from atomizer. Moreover, division of the dose into portions is not always reflected on curve of injection course. For some values of time of the gap, the needle can not be settled in the seat, and only reduced fuel outflow rate can be observed. Therefore, the differences exist and comparative analysis between the times mentioned at the beginning is justified.

In the Fig. 7 is presented comparison of preset and calculated sums of individual portions of the dose. They were arranged in groups according to preset times and shapes of injection. It can be noticed a significant differences between preset and calculated times. The differences concern all analyzed cases. The biggest difference occurred for multiportion times and higher pressure values, and amounted to $2354 \mu \mathrm{s}$. For much shorter, two-portion times and lower pressures much smaller value of the difference $-773 \mu$ s was recorded.

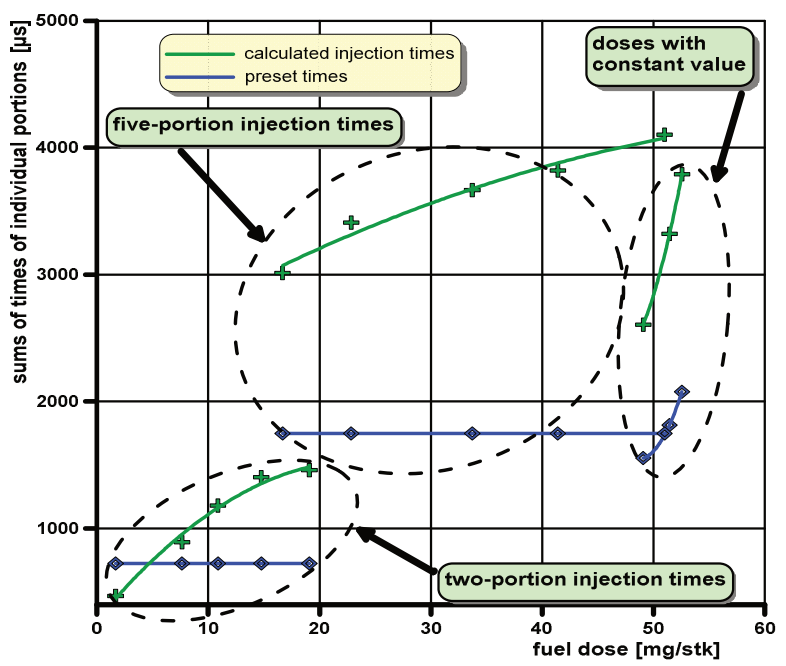

Fig. 7. Collation of sums of times of individual portions of the dose for the analyzed measuring cases

A similar comparison was performed for preset and calculated sums of total injection times. They were summarized in the Fig. 8. Here the absolute differences are lower than these analyzed above, and for five-portion times do not exceed $350 \mu \mathrm{s}$, and for two-portion $635 \mu \mathrm{s}$.

Because the analyzed quantities are characterized by considerable variability of values, in the comparative analysis were also used relative, percentage differences of analyzed times. They were calculated as quotient of difference of preset, total times of injection (preset sums of times of respective portions) and calculated, total times of injection (calculated sums of times of respective portions), related to preset, total times of injection (preset sums of times of respective portions).

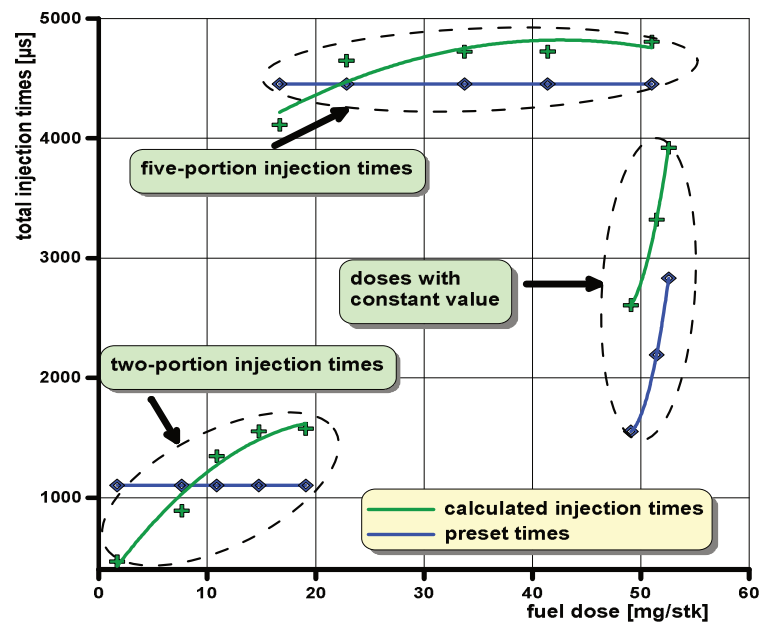

Fig. 8. Collation of sums of total times for the analyzed measuring cases 
The results in function of total dose of the fuel are presented in the Fig. 9. The dashed lines indicate changes in total times, while solid lines depict changes of sums of respective portions. Nature of the changes of these courses is similar to linear.

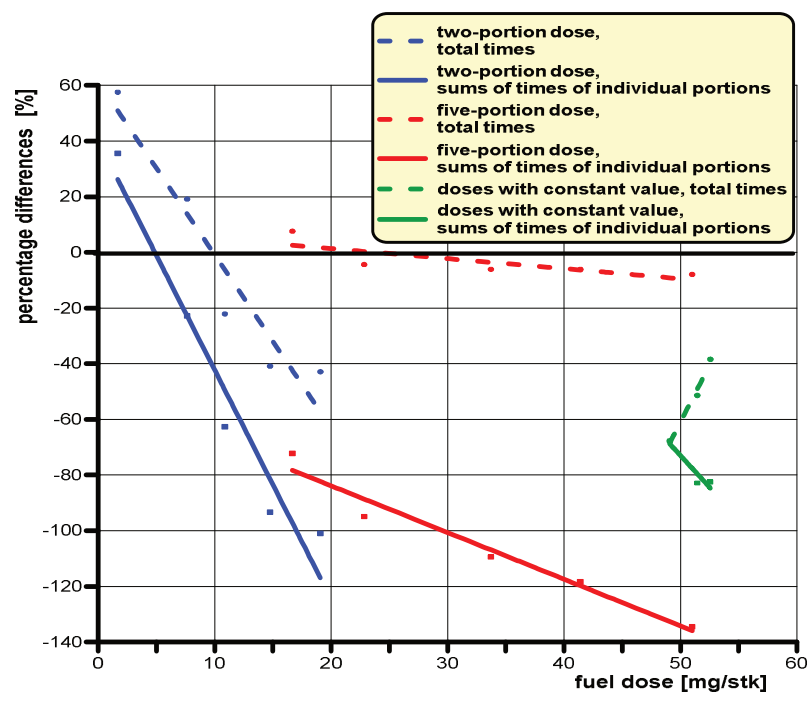

Fig. 9. Percentage differences between preset and calculated injection times, evaluated for two- and five-portion doses

As can be noticed, in majority of the cases the calculated times are greater than the preset times. Only for the short times and low pressures this regularity is not fulfilled. Distinct differences between preset and calculated times occur especially for the sum of individual portions of the dose (solid lines). In this case, values of the differences are growing rapidly as the dose increases, and for five-portion division, where percentage differences practically in complete analyzed range are bigger than $90 \%$, and even can reach up to $140 \%$. Such significant differences result mainly from the causes listed in performed earlier comparative analysis of preset injection times and times of needle lift [4], i.e. from increase rates of amperage controlling injectors, values of preset injection times of respective portions of the dose and gaps between the injections, delays in beginning and finishing of the injection. There are also others, resulting from calculations of injection course and associated necessity of interpretation of injection courses from indicator of injection dose. As already notices at beginning of the discussion, times of needle lift of individual portions of the dose were measured with arbitrarily taken assumption on beginning and ending of process of pressure growth in chamber of the device. Despite all the diligence, it is not possible to capture all small changes in course of injection which affect values of analyzed times. Process of evaluation of injection courses, which were the basis to calculation of injection times, is based on processes of approximation and differentiation of averaged, measured values. Particular attention is required in case of pressure courses obtained in result of injection of multi-portion doses. In these cases, to maintain accuracy of simulation of injection process, it is necessary to divide the courses into parts, and for each part to carefully select approximation function to differentiate it, and next reconnect the parts into a single course [7]. In particular, computation of the derivative reveals and enhances each recorded local change in the output course, and if it concerns the beginning and the ending of analyzed curve, it makes it difficult to evaluate the root-values. In these stages of the proceedings could be seen some sources of discrepancy between times of needle lift and calculated injection times, and in consequence, sources of increased percentage differences between preset and calculated injection times.

For two-portion division, when only one gap was preset, the differences are smaller, due to lower values of injection pressure and are resulting mainly from smaller values of injection pressure and connected with it - for some measuring points - lacking division of the dose into portions.

In the Fig. 9 are also presented percentage differences in preset and calculated injection times, evaluated for constant fuel dose (the second measuring group). From the course of changes of differences in sums of preset and calculated times of respective portions of the dose (solid line) is seen that in the analyzed range the sums of calculated times are bigger than the preset ones, and the differences reach $80 \%$. The highest values of the differences are present for the biggest number of divisions of the dose, while the smallest ones for a single-portion dose. When performing analyzes for this measuring group it was possible to evaluate times of the lift for respective portions of the dose (based on course of pressure in chamber of the indicator), but in case of the calculated times it wasn't possible because only significant changes in fuel outflow rate were observed, none division of the dose into portion occurred. Thus, to evaluate percentage differences in the preset and calculated times of individual portions of the dose, it was necessary to take the total calculated injection times and refer them to sums of preset times of individual portions of the dose. For this reason, discussed percentage differences have a higher values than in case of comparison of preset injection times with needle lift.

Values of percentage differences of the total times (dotted lines) differ to a small extent with analogical, plotted earlier values for times of the lift [4]. This complies with expectations, because calculated total times were evaluated on the basis of total times of the lift. Here too, although to much smaller degree, can be applied the above presented remarks concerning discrepancy between preset and calculated times. The differences are mainly due to difficulties in evaluation of zero level of calculated courses, and referencing to times of the lift, the differences do not exceed 5\%. A slight increase in total injection time is only noticeable for a bigger doses.

Verification of the calculations was comparison of the calculated unit dose of the fuel with the dose measured in testing station. In vast majority of the cases the differences between these values were included within interval 2.3$6.6 \%$. Only in two cases of very small doses were observed a higher values: 12.7 and $30.4 \%$. On this basis it was concluded that calculated curves, being the basis for analytical evaluation of size of the dose, accurately reflect duration of fuel supply process.

In the further part of the analysis consideration was given to share of calculated times of individual portions and 
gaps in total calculated injection time. The analyzes were performed for the first and the third measuring groups. The share of individual portions of the dose in total mass of supplied fuel was also taken into account. Names of individual portions of the dose were adopted according to terminology used in the English language literature, that is: the first part is called ,pilot", the second ,pre”, the third „main”, the fourth ,after” and the fifth ,post”.

And thus, in the Fig. 10, for the first measuring group and investigated values of fuel pressure in the rail, is presented percentage share of calculated times of individual portions and gaps in total, calculated injection time. Obtained values were referred to percentage share of the preset times of individual portions and gaps in total preset time of the injection. (left part of the drawing). In spite of significant shares of all preset values (the first portion - pilot $23.8 \%$, the second portion - main $-42.1 \%$, gap $-34.2 \%$ ) in the total preset injection time, from calculated courses of fuel outflow rate results that for two the lowest pressures the fuel was supplied in form of a single portion dose. For the next three values already occurred division into two portions, with domination of share of the second portion of calculated time of injection. For two the highest pressures its share was practically maintained on a constant level (about 68\%). At these points, the changes occur for the first portion and for the gap, while the first portion of the dose was extended at the expense of the gap (occurs increase of share of the first portion from $22.4 \%$ to $25.2 \%$ ).
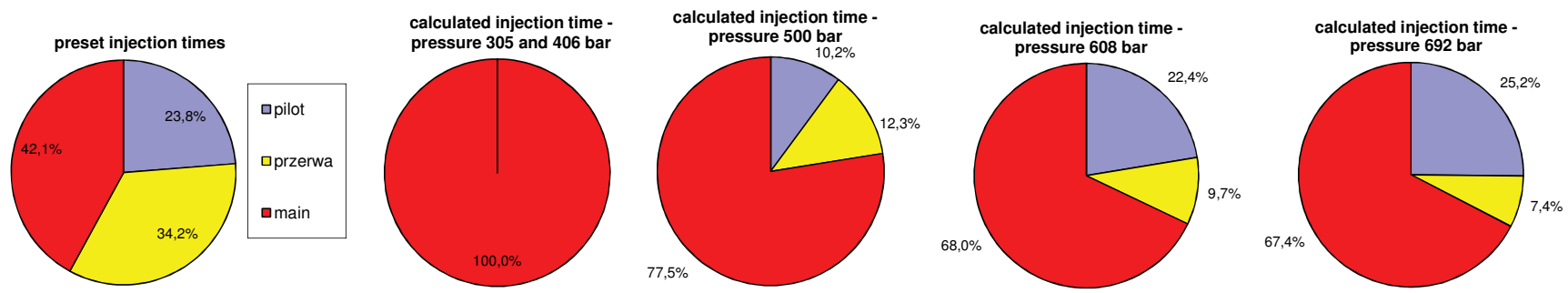

Fig. 10. Percentage shares of calculated times of individual portions and gaps in total, complete injection time for the first group of the measurements

Consequently, it would be expected a significant increase of mass of fuel supplied with the first portion of the dose. However, from summarization presented in the Fig. 11 is seen that this mass increases, but more slowly than indicated by share of the time. Thus, with increase of pressure, for the first portion of the dose is seen reported earlier increase of fuel outflow rate from the atomizer. However, it is not as much important that share of fuel mass of this portion in the total dose is comparable with share of time of this portion in total calculated time of the injection. This is insofar important observation, that when developing control algorithms of the engine (or when modeling working processes of the engine), it is important to take into considerations both calculated quantities of the fuel, evaluated for respective portions of the dose, as well as calculated times of the injection.

When injecting small doses at low injection pressures, the question arises what values of preset injection times should be used. Omitting issues connected with spraying of the fuel, based on the data presented above, it can be ascertained that divided times shouldn't be set, because division into portions will never be accomplished. However, from the point of view of need of proper and long-lasting operation of injector's valve, divided times should be used. In this way is reduced amount of energy required to controlling of the valve, and thus amount of heat generated in the valve and temperature of fuel flowing across the fuel overflow. It should be remembers, however, that times of gaps shouldn't be shorter than delay times of completion of the injection. Otherwise, process of needle lift, initiated by the first from preset portions, may be interrupted. With a higher fuel pressure, it is possible to use successfully divided times to modulate the stream of fuel supplied to the cylinder. This method seems especially effective for low and medium engine loads, where the first portion of the dose has a significant effect on level of emissions of nitrogen oxides, while the second portion on effective performance of the engine.
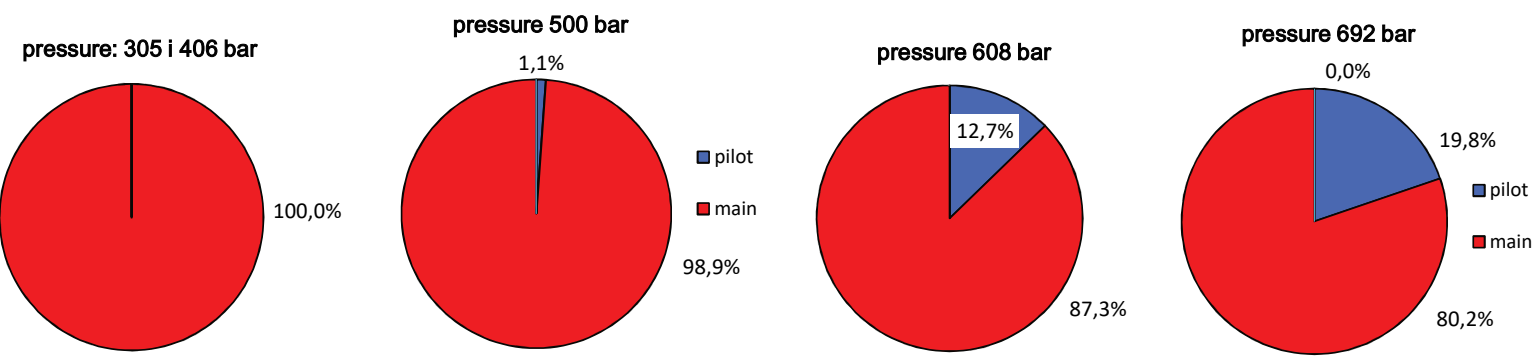

Figs. 11. Percentage share of individual portions of the dose in total amount of supplied fuel for the first group of the measurements

In turn, in the Fig 12 is shown, for the third group of the measurements and analyzed values of pressure in the rail, percentage share of calculated times of individual portions and gaps in the total calculated time of the injection. These values were compared with percentage share of preset times of individual portions and gaps, in the total preset time of injection (left part of the drawing). 
Shares of preset times are significantly smaller than shares of calculated times. In the total preset injection time the biggest share - more than $60 \%$ - belongs to gaps. For calculated times the shares are significantly smaller and decrease with increase of pressure (from $28.5 \%$ to $14.6 \%$ ). The share of the biggest portion - the main portion - increases significantly, especially when combined with Pre portion (also Fig. 12). Phenomenon of linking the portions begins to occur for fuel pressures in the rail highest than 650 bar and was discussed earlier.

When considering amounts of fuel supplied in the individual portions (Fig. 13), the third portion of the dose (main) draws the attention, as dominating the others. As the pressure increases, share of fuel supplied by this portion increases from $45 \%$ up to $58 \%$. The first portion of the dose (pilot) is the most stable. Its share amounts to about $7 \%$.

Significant effect of times of gaps on injection process is seen when multi-portion dose of injection is used. By their proper selection, various forms of injection course can be created, without significant increase in total time of the injection. It is also possible to achieve maximal value of fuel outflow rate at predominated, in respect to beginning of injection, position. Fuel pressure fluctuations do not significantly affect this position, but at the same time they allow for changing dose and spraying quality of fuel.

Generally speaking, the longest gaps promote generation of divisions into portions, but considerations should be always given to pressure level of fuel in the rail, strongly impacted on form of the injection. Design features of a given type of injector, having effect on characteristic times of injection, are also important. Mostly, it should be listed here: mass and dimensions of the atomizer's nozzle, geometrical parameters of control piston and control chamber, and operational parameters of quick-toggling valve, the valve incorporated into upper part of the injector.

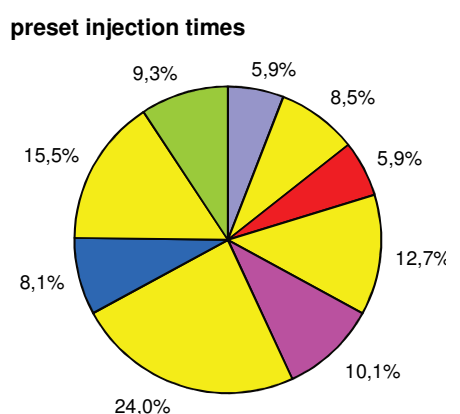

calculated injection times pressure 562 bar

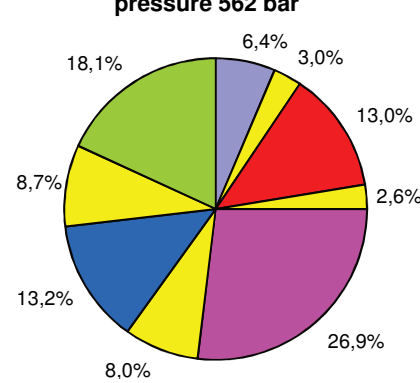

calculated injection times pressure 379 bar

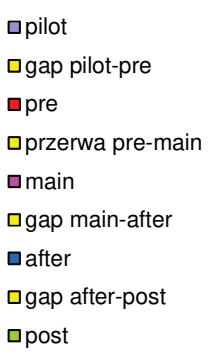

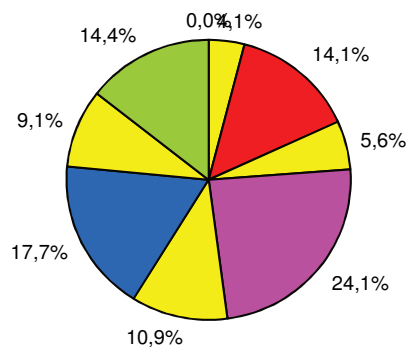

calculated injection times pressure 452 bar

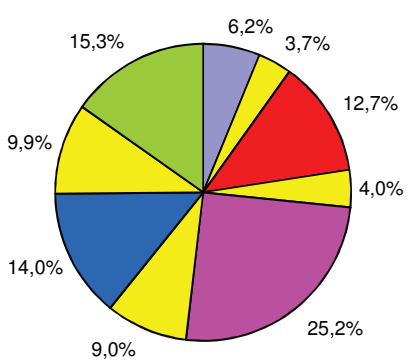

calculated injection times - pressure 648 bar

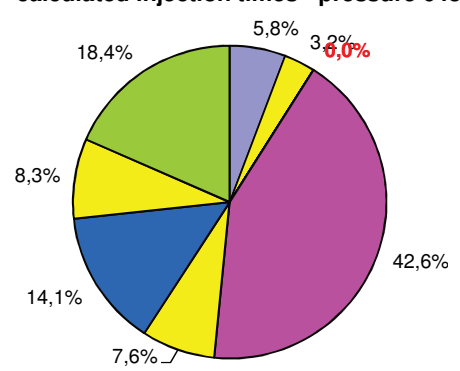

calculated injection times - pressure 764 bar

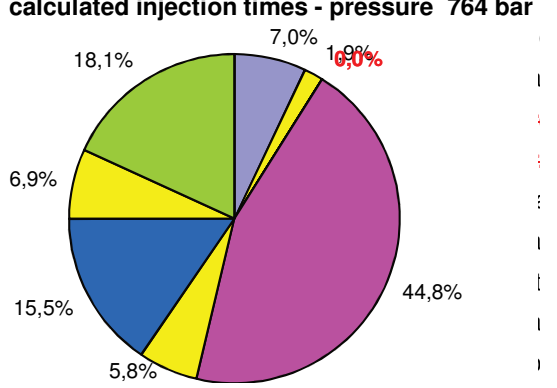

Fig. 12. Percentage shares of calculated times of individual portions and gaps in total, complete injection time for the third group of the measurements
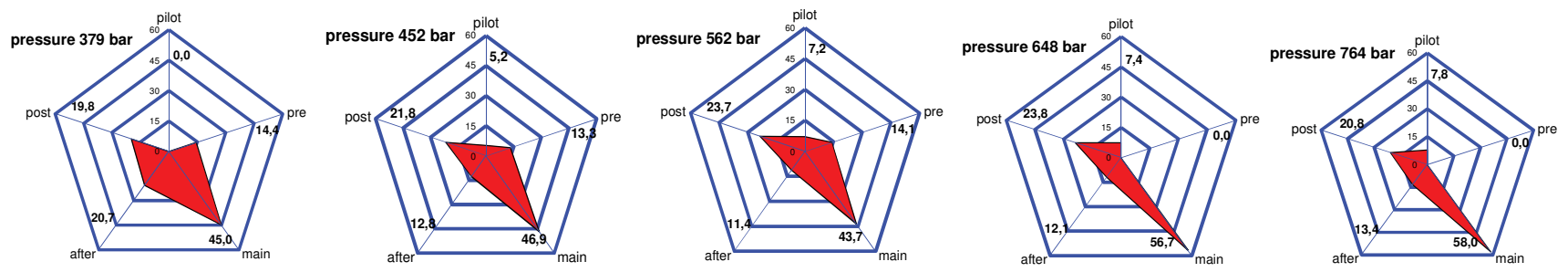

Fig. 13. Percentage share of individual portions of dose in total amount of supplied fuel for the third group of the measurements

\section{Summary}

Calculated injection times are different from preset times. This is decided by many factors, but the most important are: control method of electromagnetic valve (course control current flow), inertia of movable components of injector and pressure in high-pressure part of injection system. During injection are occurring factors causing both reduction and increase of injection time. Finally, however, the times during which the fuel is supplied are in the vast majority of cases higher than preset injection times. Exceptions are the cases where, at low fuel pressure in the rail, small doses of fuel are supplied. Therefore, for fixed surface area of outflow ports from the atomizer and fixed pressure of fuel injection the dose can not be equated with 
preset injection times. They will depend both on features adequate to a given injector (operational parameters of control valve, moving masses of the injector, viscous resistance of motion, etc.) as well as values of injection times and gaps between injections, or number of portions to which the dose was divided.

Increasing duration of fuel supply is associated with increase of times of individual doses, constituting the fuel dose. Intensity of changes in time of the process depends on values of preset parameters. Thus, increasing supply pressure of fuel results in decrease of delay time of beginning of injection and increasing of delay time of termination of injection, and thus causing a high increase of time of needle lift. Similarly are affecting two phenomena: inductivity associated with course of controlling electric current of the valve and magnetic residue of the valve, depending on preset injection time. The both ones increase delay times of injection ending. In turn, increasing the preset times of gap results in reduced delay times of injection ending and reduced time of needle lift. However, its effect is smaller than the others. As a result, injection times of individual portions, and the same entire injection cycle, are prolonged.

Clearly, the intervals between dose portions disappear. This phenomenon has an effect on the mass of fuel that is given in the individual parts of the dose. The share of fuel masses in the individual dose portions is greater than that calculated from the calculated injection times (differences up to $25 \%$ ).

The knowledge of calculated injection times is one of elements that allow development of control algorithms to compression ignition engines. It allows significant reduction of labour consumption needed for application of software in controller of the power unit. The results of performed comparative analysis of preset and calculated injection times may be used to calculation of energy stream supplied to combustion chamber during individual phases of the process. They can be also helpful in determining characteristic parameters of the injection, such as maximal injection pressure and fuel outflow from atomizer.

It should be remembered that use of multi-portion doses of the injection doesn't result in any significant increase in total, calculated values of injection time relative to the preset ones. However, under certain circumstances, calculated values of the times are significantly increased. This occurs when a small unit dose of fuel is supplied, and when high values of preset times are used. Calculated durations of individual portions of the dose are much longer than their preset counterparts. The differences are due to the same reasons, as these described during comparison of preset times with lift of the needle, and additionally are coming here discrepancies connected with transformations of measured coursed into smooth curves and resulted from their differentiation.

Division of fuel dose into portions can be accomplished under certain conditions. First and foremost, a sufficiently high fuel pressure should be generated and sufficiently long times of gaps should be set. When are set constant values of injection time, times of needle lift for individual portions of the dose increase together with increase of pressure. Such increase depends on size of the gap, because for longer gaps the delay time of injection ending increases, and the same time of the lift. This in turn reduces gaps.

Presented analyses concerning the processes governing injection times, starting from presetting in the controller up to moment of execution in compression ignition engine, could be a tool for research engineers, writing or modifying control algorithms to the power units. The comparisons, specifications and dependencies presented in this paper allow considerable reduction of time spent on work connected with selection and calibration of fuel supply system.

\section{Bibliography}

[1] BINGQI, T., LIYUN, F., XIUZHEN, M. et al. Investigation of main injection quantity fluctuation due to pilot injection in high pressure common rail fuel injection system. International Journal on Smart Sensing and Intelligent Systems. 2014, 7(2), 820-836

[2] HERFATMANESH, M. R., PENG, Z., IHRACSKA, A. et al. Characteristics of pressure wave in common rail fuel injection system of high-speed direct injection diesel engines. Advances in Mechanical Engineering. 2016, 8(5), 1-8.

[3] KNEFEL, T. The evaluation and interpretation of the characteristic injection times of a multiple fuel dose. Journal of KONES: Powertrain and Transport. 2011, 18(2), 205-213.

[4] KNEFEL, T. Analiza porównawcza wybranych czasów wtrysku wieloczęściowej dawki paliwa. Czasopismo Techniczne. 2012, 9, 115-129.

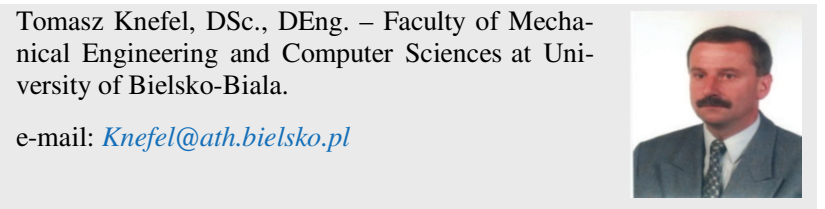

[5] STELMASIAK, Z., KNEFEL, T., LARISCH, J. A development control unit for common rail injection system KSSiP-1. Combustion Engines. 2005.

[6] KNEFEL, T. Electronically controlled indicator of injection. Combustion Engines. 2007, SC2, 74-79.

[7] KNEFEL, T. Methodology of determination diesel engine injection rate and dose with the assistance indicator of injection. Combustion Engines. 2009, SC2, 379-385.

[8] WANG, X., KIKUTANI, T., TAKEUCHI, K., NAKANE, N. Development toward "diesel revolution" using ultra high pressure CRS with closed-loop control system for heavy duty engine. FISITA World Automotive Congress, 2010, Budapest, F2010-A-164.

Jacek Nowakowski, DSc., DEng. - Faculty of Mechanical Engineering and Computer Sciences at University of Bielsko-Biala.

e-mail:JNowakow@ath.bielsko.pl 